An integrated interface circuit with a capacitance-to-voltage converter as front-end for grounded capacitive sensors

This article has been downloaded from IOPscience. Please scroll down to see the full text article.

2009 Meas. Sci. Technol. 20015202

(http://iopscience.iop.org/0957-0233/20/1/015202)

View the table of contents for this issue, or go to the journal homepage for more

Download details:

IP Address: 131.180.130.109

The article was downloaded on 08/08/2011 at 10:40

Please note that terms and conditions apply. 


\title{
An integrated interface circuit with a capacitance-to-voltage converter as front-end for grounded capacitive sensors
}

\author{
Ali Heidary and Gerard C M Meijer \\ Electronic Instrumentation Laboratory, Delft University of Technology (TU Delft), Mekelweg 4, \\ 2628 CD Delft, The Netherlands \\ E-mail: a.heidary@tudelft.nl
}

Received 25 May 2008, in final form 11 September 2008

Published 12 November 2008

Online at stacks.iop.org/MST/20/015202

\begin{abstract}
This paper presents the analysis and design of an integrated interface for grounded capacitive sensors. To reduce the effects of parasitic cable capacitances, a feedforward technique has been applied. In combination with the use of a special front-end amplifier this yields high immunity for a parasitic cable capacitance. The major nonidealities of the interface circuit have been analyzed. The complete interface has been designed and implemented as an integrated circuit, using standard $0.7 \mu \mathrm{m}$ CMOS technology. Experimental results, which are in good agreement with theoretical analysis and simulation, show that for sensor capacitance down to $10 \mathrm{pF}$, shielded connection cables up to $30 \mathrm{~m}$ can be handled with an absolute error of less than $0.3 \mathrm{pF}$. The measured nonlinearity of the interface amounts to about $3 \times 10^{-4}$ for $30 \mathrm{~m}$ of the cable. For $40 \mathrm{~ms}$ measurement time, the resolution amounts to about 16 bits.
\end{abstract}

Keywords: active shielding, capacitance-to-voltage converter, capacitive sensor, integrated interface

(Some figures in this article are in colour only in the electronic version)

\section{Introduction}

Capacitive sensors are widely applied in, for instance, liquidlevel gauges, pressure meters, accelerometers and mechanical high-precision positioners. In such applications, physical or mechanical quantities are converted into capacitance values, which are further processed by an electronic circuit, the modifier. Often, capacitive sensor elements are connected to the electronic interface circuitry with long wires of cables. To reduce the effects of interference, these connecting wires or cables are shielded. Provisions have to be taken to avoid the parasitic capacitances of these cables forming direct shunting components for the sensing elements because without such provisions any changes in these parasitic capacitances would seriously degrade the sensor-system performance.

When the capacitive sensor elements are floating, i.e. when none of the terminals has been connected to the ground, then they can be read by interface circuits that are intrinsically immune to stray capacitances to the ground [1]. Also it is possible to do two-step measurements in order to extract the value of a floating capacitance independent of the parasitic capacitance to the ground [2]. However, safety reasons and/or operating limitations might require that one of the electrodes of the sensing elements be grounded. This is the case, for instance, with level measurement of a conductive liquid in a grounded metallic container with a capacitive sensor [3, 4].

For grounded capacitive sensors, a usual way to reduce the effects of shunting parasitic capacitances is to apply active shielding (figure 1) [1]. In figure $1, C_{\mathrm{p} 1}$ and $C_{\mathrm{p} 2}$ represent the capacitance between the core conductors of the coaxial cable with its shield and the capacitance of the shield to the ground, respectively.

There is a trade-off between the accuracy and the stability of this system. Especially, when the values of parasitic capacitances are not known or can vary over a wide range, it is difficult to optimize the system for its performance [5]. To solve this problem, in a recent publication [6], a novel interface has been introduced in which active shields have been 


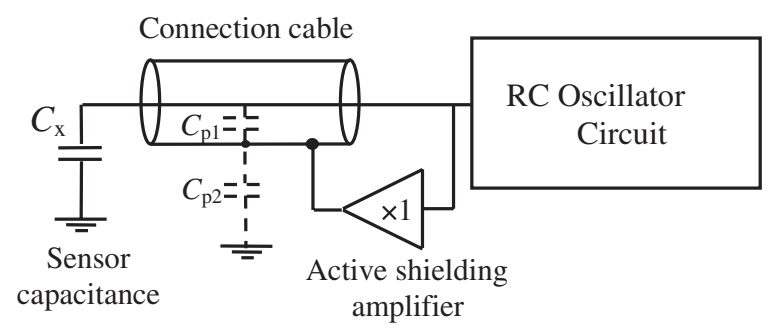

Figure 1. The usual read-out interface for the grounded capacitive sensor.

connected to a buffer voltage while using feedforward instead of feedback. It has been shown that with this technique there will not be any instability problems and there will be more design freedom to increase the accuracy.

However, the circuit described in [6] has been implemented with discrete components, and the capacitive sensing elements are directly connected to a capacitanceto-time converter. In the present paper, we will show that the use of a capacitance-to-voltage converter as front-end will significantly improve the system performance. The major nonidealities of the interface system will be discussed together with methods for reducing their influence. An integrated version of the improved interface for grounded capacitive sensor with feedforward-based active shielding will be presented. In order to reduce the effect of any low-frequency disturbing signals, including flicker noise, interference from the mains, and offset, the interface is equipped with a special kind of chopper, according to the $\left(+-+_{-}\right)$principle described in [7, 8]. Moreover, to remove any additive and multiplicative errors, which are mainly caused by uncertainty in design parameters and thermal drift, a three-signal auto-calibration technique [7] has been used. The interface has been designed and implemented using $0.7 \mu \mathrm{m}$ standard CMOS technology. The experimental results are presented in section 4. As compared to previous work [6], these results demonstrate a significant improvement in immunity for parasitic capacitances, and a higher flexibility in adapting the front end to the maximum value of sensor capacitances.

\section{System setup and front-end circuit}

Figure 2(a) shows the complete setup, which consists of a multiplexer, a new capacitance-to-voltage converter, a voltageto-period converter $[6,7]$ and a control unit. The output signal is shown in figure $2(b)$. According to the use of the three-signal auto-calibration technique [8], one measurement cycle consists of three phases in which a first reference capacitor $C_{\text {ref1 }}$, a second reference capacitor $C_{\text {ref2 }}$ and the sensor capacitor $C_{x}$ are measured, respectively. Their values are linearly converted to the time domain and result in corresponding time periods $T_{\text {ref1 }}$, $T_{\text {ref2 }}$ and $T_{x}$ of the output signal. For identification purposes, the time interval $T_{\text {ref1 }}$ is split into two short periods [8]. For the three time intervals it holds that

$$
T_{\text {ref1 }}=a C_{\text {ref } 1}+b
$$

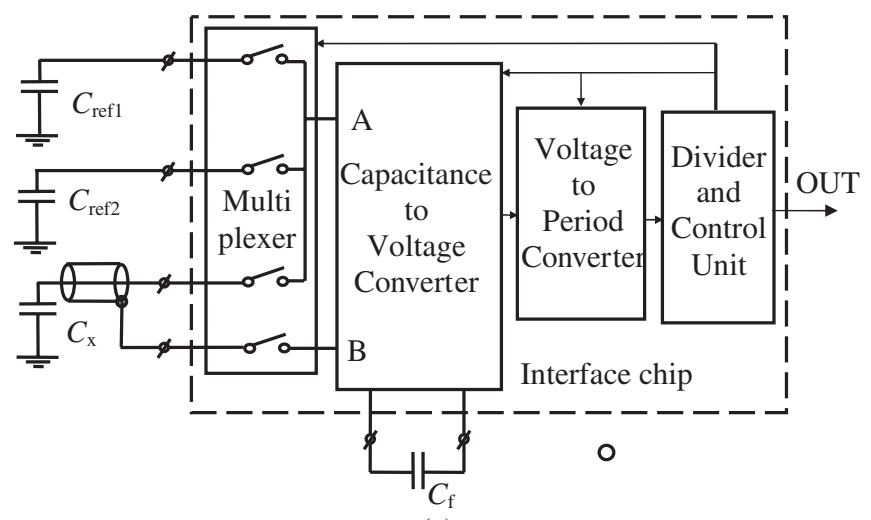

(a)

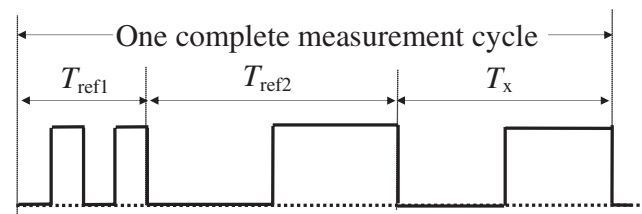

(b)

Figure 2. (a) The complete interface including three-signal auto-calibration and $(b)$ the interface output signal.

$$
\begin{aligned}
T_{\mathrm{ref} 2} & =a C_{\mathrm{ref} 2}+b, \\
T_{x} & =a C_{x}+b,
\end{aligned}
$$

where $a$ and $b$ represent the multiplicative and additive parameters of the capacitance-to-time converter. After measuring the length of the different periods with a microcontroller, the value of a parameter $M$ is calculated using the equation

$$
M=\frac{T_{x}-T_{\text {ref1 }}}{T_{\text {ref2 }}-T_{\text {ref1 }}}=\frac{C_{x}-C_{\text {ref1 }}}{C_{\text {ref } 2}-C_{\text {ref } 1}} .
$$

From this value and supposing that the values of $C_{\text {ref1 }}$ and $C_{\text {ref2 }}$ are known, the input capacitance $C_{x}$ can be extracted. From equation (4) it can be concluded that $M$ and therefore also the measured value of $C_{x}$ are independent of $a$ and $b$. Therefore, the measurement result is independent of any changes of the additive and multiplicative parameters of the interface circuit, as can be caused by process spread, temperature, and so on.

To get a good resolution, the difference $\left(C_{\text {ref2 }}-C_{\text {ref1 }}\right)$ between the values of the reference capacitors should be large enough. On the other hand, as will be explained in section 3.3, the values of $C_{\text {ref1 }}$ and $C_{\text {ref2 }}$ should be chosen in such a way that the interface circuit will work in its linear region.

Figure 3(a) shows the capacitor-to-voltage converter, for the case that the sensor capacitor $C_{x}$ is selected. The switch pairs, $\left(S_{1}, S_{2}\right),\left(S_{2}, S_{4}\right)$ and $\left(S_{2}, S_{3}\right)$, all work in a break-beforemake mode. This will guarantee that no charge is lost at the negative input of the amplifier. To understand how this SC circuit works, we first suppose that the cable capacitances $C_{\mathrm{p} 1}$ and $C_{\mathrm{p} 2}$ are zero and that the amplifier $\mathrm{A}_{1}$ and the switches are ideal.

During time interval $T_{1}$ (figure $3(b)$ ), $\mathrm{S}_{1}$ is $\mathrm{ON}$ which sets $V_{\text {out }}$ to $V_{\mathrm{dd}} / 2$. At the same time, via $\mathrm{S}_{3}$, the top electrode of the sensor capacitance $C_{x}$ is connected to the ground. During time interval $T_{2}, C_{x}$ is connected to the negative input of the 


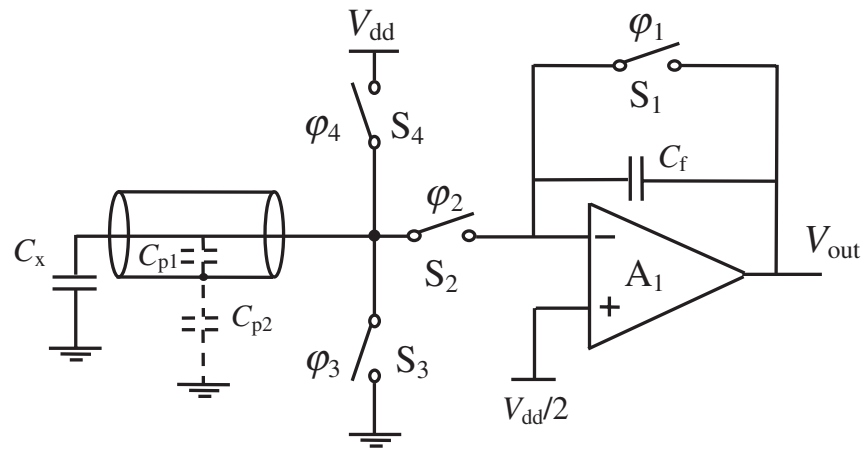

(a)

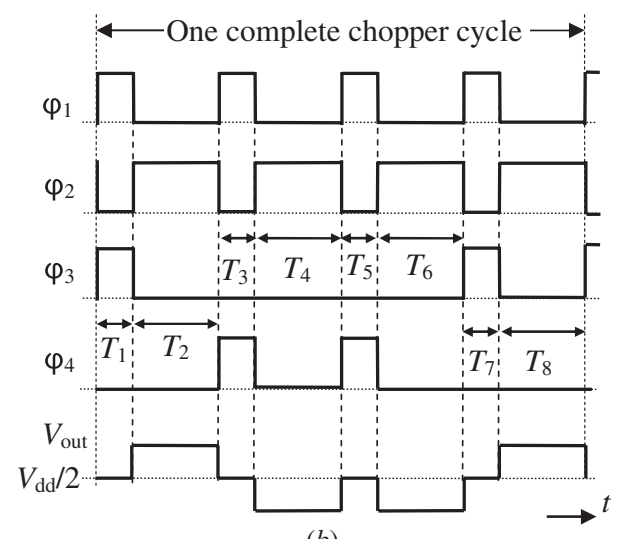

(b)

Figure 3. (a) The new front-end for the grounded capacitive sensor and $(b)$ the switch-control signals and the output voltage.

amplifier. As a consequence, a charge $C_{x} V_{\mathrm{dd}} / 2$ will be pumped to $C_{f}$, which results in a jump $C_{x} V_{\mathrm{dd}} /\left(2 C_{f}\right)$ of the output voltage $V_{\text {out }}$. In a similar way, for the other time intervals, the value of $V_{\text {out }}$ can be found, as depicted in figure $3(b)$.

In the setup of figure $3(a)$, the excitation voltage for the capacitor $C_{x}$ can have one of three well-known values: $0 \mathrm{~V}, V_{\mathrm{dd}}$ and $V_{\mathrm{dd}} / 2$. Knowing this in advance, without using feedback, we can apply the same voltage to the shielding conductor. In this way, the effect of cable parasitic capacitances can be eliminated without having instability problem.

In our design, we need to cover sensor capacitances up to $330 \mathrm{pF}$. In such a case $C_{f}$ will be too big to be integrated. Therefore, for the capacitor $C_{f}$ we used an off-chip component. The value of this capacitor can be optimized to obtain the maximum output swing of the amplifier for the maximum value of $C_{x}$. Next, the following stage, the voltage-to-period converter, can be optimized independently of the sensorcapacitance range. This also allows the end user to optimize the system performance for his specific application.

\section{Effects of component imperfections}

The major nonidealities are the amplifier offset, the switchcharge injection and the switch $\mathrm{ON}$ resistance. In this section the influence of these nonidealities will be discussed.

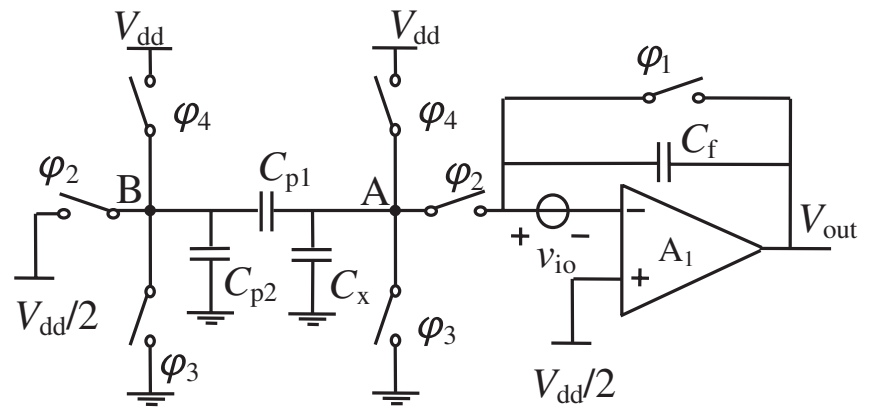

(a)

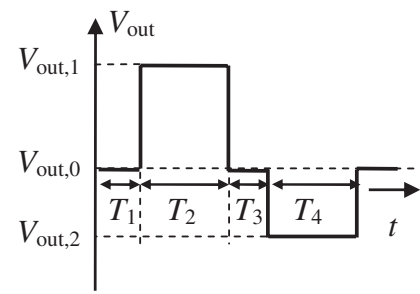

(b)

Figure 4. (a) The new front-end with shield driver and $(b)$ the output voltage at the presence of offset.

\subsection{The offset}

Figure 4(a) shows the front-end circuit for the case that the sensor-cable shield (point B) is driven with the same voltage as the cable core (point A). If we suppose that the switches and the voltage source are ideal, then $C_{\mathrm{p} 2}$ cannot play any role.

The main objective of active shielding is keeping the voltage across $C_{\mathrm{p} 1}$ at zero, but when $\varphi_{2}$ is high, the voltage across $C_{\mathrm{p} 1}$ equals the input offset voltage $v_{\text {io }}$ of the amplifier. The effect of this offset voltage is eliminated by the applied chopper, as will be shown now:

During time interval $T_{1}$ (figure $4(b)$ ), $V_{\text {out }}$ will be set to $V_{\text {out }, 0}=V_{\text {dd }} / 2+v_{\text {io }}$. At the same time, the top electrode of the sensor and the shield are connected to the ground. During time interval $T_{2}$ a charge $q_{1}$, which equals

$$
q_{1}=C_{x} V_{\mathrm{dd}} / 2+\left(C_{x}+C_{\mathrm{p} 1}\right) v_{\mathrm{io}}
$$

will be pumped into $C_{f}$. This will result in an output voltage $V_{\text {out }, 1}$, which equals:

$$
V_{\text {out }, 1}=V_{\text {out }, 0}+\frac{C_{x} V_{\mathrm{dd}} / 2+\left(C_{x}+C_{\mathrm{p} 1}\right) v_{\text {io }}}{C_{f}} .
$$

In a similar way we will have

$$
V_{\text {out }, 2}=V_{\text {out }, 0}+\frac{-C_{x} V_{\mathrm{dd}} / 2+\left(C_{x}+C_{\mathrm{p} 1}\right) v_{\mathrm{io}}}{C_{f}} .
$$

As we can see, due to the offset, the output voltage will not be symmetrical with respect to the level of $V_{\text {out }, 0}$, anymore. However, the next stage, which is a voltage-to-period converter $[5,6]$, is designed to be only sensitive to the peak-to-peak voltage $V_{\mathrm{p}-\mathrm{p}}$, which equals

$$
V_{\mathrm{p}-\mathrm{p}}=\left(V_{\text {out }, 1}-V_{\text {out }, 2}\right)=\frac{C_{x} V_{\mathrm{dd}}}{C_{f}},
$$

and is independent of offset. 


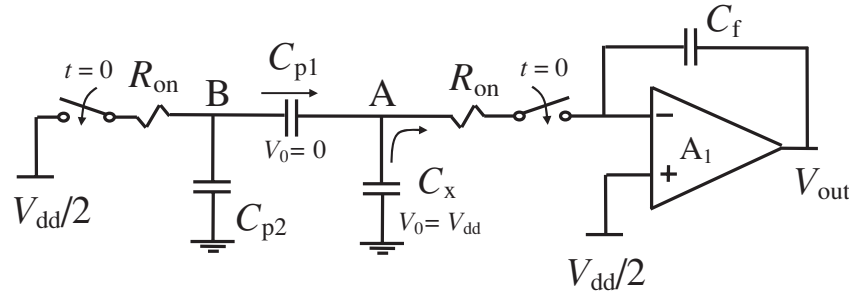

Figure 5. The relevant part of the interface to analyze the charge transfer process.

\subsection{Switch ON resistance, $R_{\text {on }}$}

Figure 5 shows the circuit of figure 4 at the beginning of the time interval $T_{4}$ in which the capacitor $C_{\mathrm{x}}$ has been charged to $V_{\mathrm{dd}}$, and that this charge is going to be transferred to $C_{f}$. Since the points $\mathrm{A}$ and $\mathrm{B}$ are switched together to the same potential, after settling, the final charge of the parasitic capacitance $C_{\mathrm{p} 1}$ will be zero. Thus, all extra charge $C_{x} V_{\mathrm{dd}} / 2$ of $C_{x}$ has been transferred to $C_{f}$.

The range of the different capacitors in this circuit and also the switch size will determine the charge transfer speed and, in combination with the available time, also the accuracy of this transfer. If we use a coaxial cable, the safety ground could be used as the return path. However, in that case the current loop will be too big and undefined, and therefore susceptible to interference. It is better to use another wire to connect the ground of the capacitive sensor, which is implemented using a special ground electrode, to the ground of the interface. The best option is to use a triaxial cable instead of a coaxial cable. Then $C_{\mathrm{p} 2}$ can be in the same range as $C_{\mathrm{p} 1}\left(100 \mathrm{pF} \mathrm{m}^{-1}\right)$ or even larger. Usually in a low-cost system, a single wire twisted to the coaxial cable is used as the ground. In this case $C_{\mathrm{p} 2}$ will not be well defined.

In our setup, using a coaxial cable with a surrounding twisted wire as the ground, $C_{\mathrm{p} 2}$ is about $35 \mathrm{pF} \mathrm{m}^{-1}$. To get an idea of the effect of the parasitic capacitances, we assume that the cable length $l=40 \mathrm{~m}$, so that $C_{\mathrm{p} 1}=4 \mathrm{nF}$ and $C_{\mathrm{p} 2} \approx$ $1.4 \mathrm{nF}$. Furthermore, we suppose that the sensor capacitance $C_{x}$ ranges from $10 \mathrm{pF}$ to $330 \mathrm{pF}$ and the switches are equal. In this case, the voltage transition at node $\mathrm{B}$ happens at a slower pace than that at node $\mathrm{A}$. This means that, initially, $C_{\mathrm{p} 1}$ will pump some charge into $C_{f}$ in the same direction as $C_{x}$. Next, this undesired charge is removed with a time constant of about $\tau \approx 2 R_{\mathrm{on}} C_{\mathrm{p} 1}$. Figure 6 shows the simulation result for the case that $C_{\mathrm{p} 1}=4 \mathrm{nF}, C_{\mathrm{p} 2}=1.4 \mathrm{nF}, C_{f}=1 \mathrm{nF}$ and $C_{x}=100 \mathrm{pF}$. If we suppose that this undesired charge is $k$ times as large as the desired charge $V_{\mathrm{dd}} C_{\mathrm{x}} / 2$ (see the right-hand side of figure 6), then the output voltage can be written as

$$
V_{\text {out }}(t)=\frac{V_{\mathrm{DD}} C_{x}}{2 C_{f}}\left(1+k \mathrm{e}^{-t / \tau}\right) .
$$

Therefore, the absolute error $\Delta V_{\text {out }}$ at the end of a time interval $T_{\mathrm{a}}$ amounts to

$$
\Delta V_{\text {out }}=k \frac{V_{\mathrm{DD}} C_{x}}{2 C_{f}} \mathrm{e}^{-T_{a} / \tau},
$$

where $T_{\mathrm{a}}=T_{2}=T_{4}=T_{6}=T_{8}$ is the available time for charge transfer (figure 6).

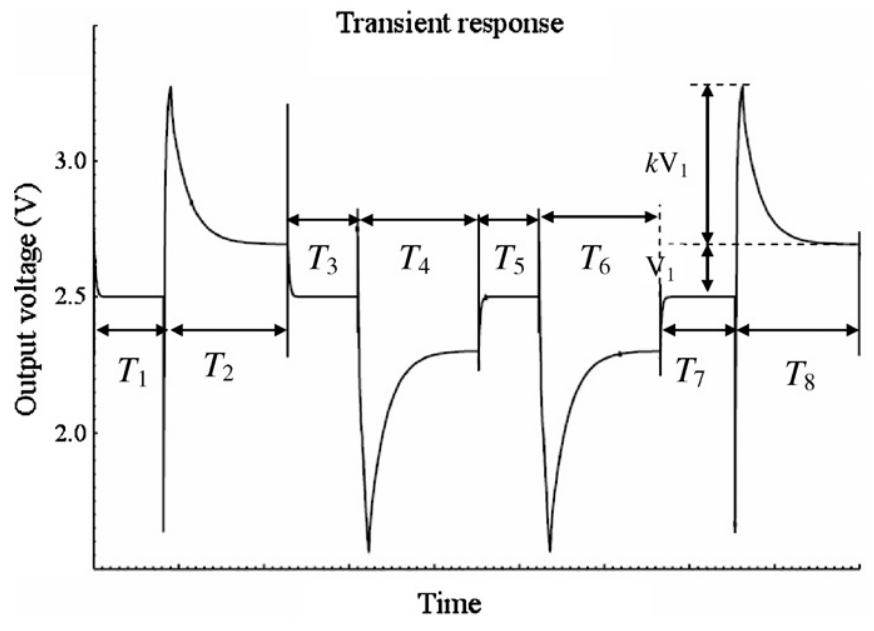

Figure 6. The transient simulation of the front-end circuit for $C_{\mathrm{p} 1}=$ $4 \mathrm{nF}, C_{\mathrm{p} 2}=1.4 \mathrm{nF}, C_{f}=1 \mathrm{nF}$ and $C_{x}=100 \mathrm{pF}$.

Translating this error to capacitance read-out error results to

$$
\varepsilon_{\text {C.T. }}=k C_{x} \mathrm{e}^{-T_{a} / \tau},
$$

where $\tau$ is the charge-transfer time constant mentioned in section 3.2. At first look, it might seem that by increasing the sensor capacitance, $C_{x}$, this error will increase too. However, in the assumed range of $C_{x}$, from $10 \mathrm{pF}$ to $330 \mathrm{pF}$, the amount of undesired charge is almost independent of $C_{x}$. As a consequence, the value of $k$ is almost proportional to $1 / C_{x}$. For instance, for $C_{\mathrm{p} 1}=4 \mathrm{nF}$ and $C_{\mathrm{p} 2}=1.4 \mathrm{nF}$ it is found (by simulation) that $k=47,10$ and 4 for $C_{x}=10 \mathrm{pF}$, $50 \mathrm{pF}$ and $100 \mathrm{pF}$, respectively. Therefore, in equation (7), for the most part the sensitivities for the parameters $k$ and $C_{\mathrm{x}}$ are compensating each other. Finally, since $T_{\mathrm{a}}$ increases with increasing $C_{x}$ [7] this error should decrease by increasing $C_{x}$, which is in agreement with the measurement result presented in section 4.

In the interface circuit, we added the option of increasing $T_{\mathrm{a}}$ by the factor of 2 , by decreasing the integrator current $I_{\text {int }}$ in the voltage-to-period converter [6]. This option can be set with a pin called slow/fast mode. For the same error, in the slow mode the chip can handle twice as long a cable than in the fast mode. As an alternative, instead of increasing the available time $T_{\mathrm{a}}$, we could also decrease the time constant $\tau$ by increasing the switch size by the same factor.

\subsection{Switch charge injection}

In order to be able to drive a parasitic cable capacitance of, for instance, $4 \mathrm{nF}$ and yet have a short settling time, we need quite big switches. Consequently, the switch-charge injection, which includes channel-charge injection and clock feed-through, [9], can be significant. Figure 7 shows the relevant part of the interface for analyzing this effect.

The charge injection of $S_{2}$ will not induce any error in the output voltage because after it turns off $\mathrm{S}_{1}$ turns on and $C_{\text {in }}$ will be connected to a well-defined potential. The error induced by charge injection of $S_{1}$ in the output voltage is always in one direction and similar to that of the offset voltage. This effect is 


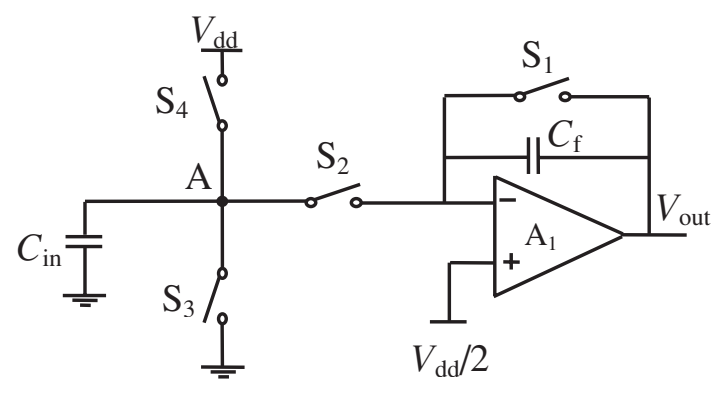

Figure 7. The relevant part of the CVC to analyze the charge-injection effect.

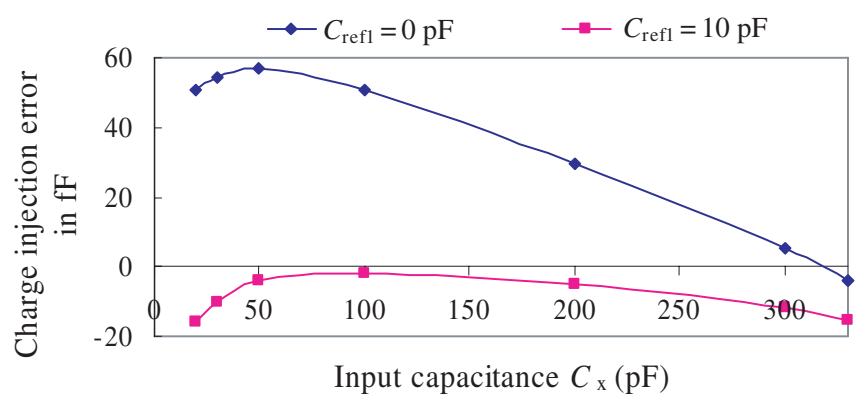

Figure 8. Simulated charge-injection-related error, $\left(C_{x, \text { cal. }}-C_{x}\right)$, versus $C_{x}$ for $C_{\text {ref } 2}=330 \mathrm{pF}$ and for $\mathrm{C}_{\text {ref } 1}=0 \mathrm{pF}$ and $10 \mathrm{pF}$, respectively.

removed by the applied chopper. However, the charge-induced errors caused by $\mathrm{S}_{3}$ and $\mathrm{S}_{4}$ will add up and are significant. For the three time periods $T_{\text {ref1 }}, T_{\text {ref2 } 2}$ and $T_{x}$ of the output signal, these errors are almost equal. Therefore, these errors will mainly be removed by applying the three-signal autocalibration technique. However, since the $C_{\text {in }}$ values (figure 7) are different for the three different phases of the measurements, the injected charges will show slight differences [9] so that after the three-signal auto-calibration some residual error remains. The largest error is found for the smallest value of $C_{\text {in. }}$.

We simulated the effect of switch-charge injection for the complete interface with $C_{\text {ref2 }}=330 \mathrm{pF}$ and $20 \mathrm{pF} \leqslant C_{\mathrm{x}} \leqslant$ $330 \mathrm{pF}$ for two values of $\mathrm{C}_{\mathrm{ref} 1}: C_{\text {ref1 }}=0 \mathrm{pF}$ and $C_{\text {ref1 }}=$ $10 \mathrm{pF}$. In order to analyze this effect independently from the error related to incomplete charge transfer, we used $C_{\mathrm{p} 1}=$ $C_{\mathrm{p} 2}=0 \mathrm{pF}$. It can be proven that parasitic capacitances $C_{\mathrm{p} 1}$ and $C_{\mathrm{p} 2}$ do not affect the switch-charge injection. Figure 8 shows the absolute error $\left(C_{x, \text { cal. }}-C_{x}\right)$ caused by charge-injection versus $C_{x}$. The value of $C_{x, \text { cal. }}$ is calculated using the equation [7]

$$
C_{\int x, \mathrm{cal}}=\left(\frac{T_{x}-T_{\text {ref1 }}}{T_{\text {ref2 }}-T_{\text {ref1 }}}\right)\left(C_{\text {ref2 }}-C_{\text {ref1 }}\right)+C_{\text {ref1 }} .
$$

From this figure, it can be seen that the residual error due to switch-charge injection can also be significantly reduced by increasing $C_{\text {ref1 }}$ to, for instance, $10 \mathrm{pF}$. As we will see in the next section, even with $C_{\text {ref1 }}=0 \mathrm{pF}$, for a long cable the error due to incomplete settling of the circuit is much larger than that caused by switch-charge injection. Therefore, for a long cable, we can simply select $C_{\text {ref1 }}=0 \mathrm{pF}$, without introducing a significant error. However, for a short cable, depending

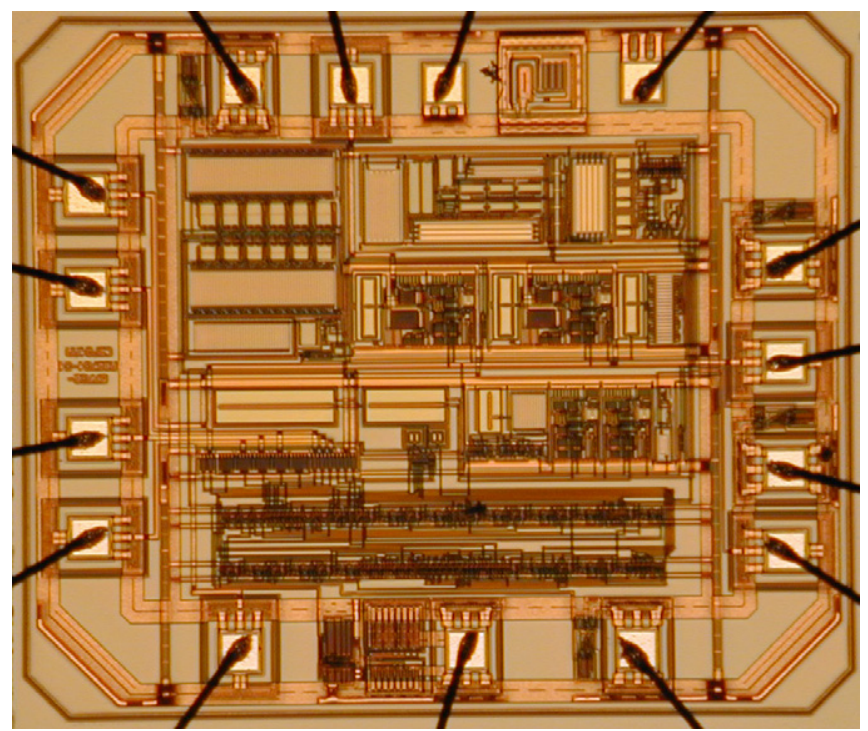

Figure 9. Photograph of the chip which measures $1.4 \mathrm{~mm} \times 1.7 \mathrm{~mm}$.

on the target accuracy, it could be advisable to select $C_{\text {ref1 }}=$ $10 \mathrm{pF}$.

\section{Experimental results}

The interface has been designed and implemented using $0.7 \mu \mathrm{m}$ standard CMOS technology. Figure 9 shows the chip photograph. The supply voltage is $5 \mathrm{~V}$ and the measured value for the supply current is about $0.7 \mathrm{~mA}$. The current consumption slightly depends on $C_{\mathrm{p} 2}$ and will increase to $0.8 \mathrm{~mA}$ for $C_{\mathrm{p} 2}=3.3 \mathrm{nF}$.

In order to see the effect of incomplete settling, we measured different capacitors from $10 \mathrm{pF}$ to $330 \mathrm{pF}$ in the fast mode for two cases: $(a)$ with emulation of $30 \mathrm{~m}$ of the coaxial cable with a twisted ground wire with equivalent discrete capacitors $C_{\mathrm{p} 1}=3 \mathrm{nF}$ and $C_{\mathrm{p} 2}=1 \mathrm{nF}$, and $(b)$ with a real cable of $30 \mathrm{~m}$ length with a twisted ground wire. The absolute error of these measurements along with the simulation result for $C_{\mathrm{p} 1}=3 \mathrm{nF}$ and $C_{\mathrm{p} 2}=1 \mathrm{nF}$ is shown in figure 10 . It can be concluded that the simulation results and measurement results for an emulated cable, with the same parasitic capacitance, are in close agreement. For a real cable, the error is a little larger. Our investigations showed that this increased error was caused by frequency-dependent leakage of the cable shield to the grounded conductor.

Figure 11 shows a comparison of the error versus the input capacitance for the slow and fast modes (section 3.2) for a real cable with a length of $30 \mathrm{~m}$. In the fast mode, the main source of error is due to uncompleted charge transfer.

Figure 12 shows the measured absolute error versus the input capacitance $C_{x}$ for four different lengths of the cable up to $40 \mathrm{~m}$ in the slow mode. From figure 12 it is easy to compare the results of the interface system presented in this paper with those of previous work [6]. According to this figure, for a sensor capacitance of $27 \mathrm{pF}$ and a cable length of $30 \mathrm{~m}$, the absolute error is about $0.25 \mathrm{pF}$. With comparable parameters, the system presented in [6] shows an error of more than $26 \mathrm{pF}$. 


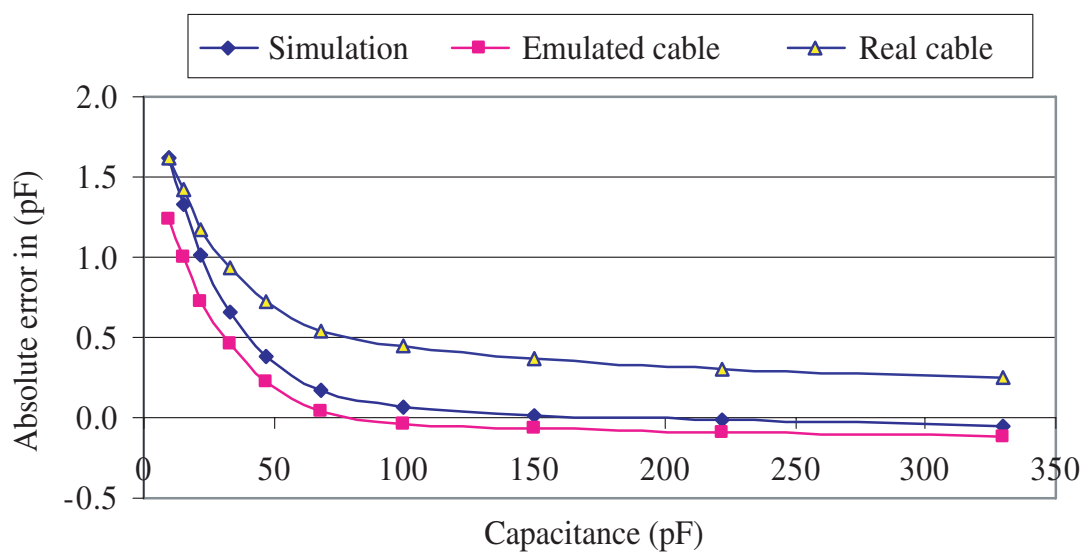

Figure 10. The simulated and measured absolute error versus the input capacitance for $30 \mathrm{~m}$ of the cable. The measurement results have been obtained with a real cable, or with emulation using equivalent capacitances $\left(C_{\mathrm{p} 1}=3 \mathrm{nF}\right.$ and $\left.C_{\mathrm{p} 2}=1 \mathrm{nF}\right)$, respectively.

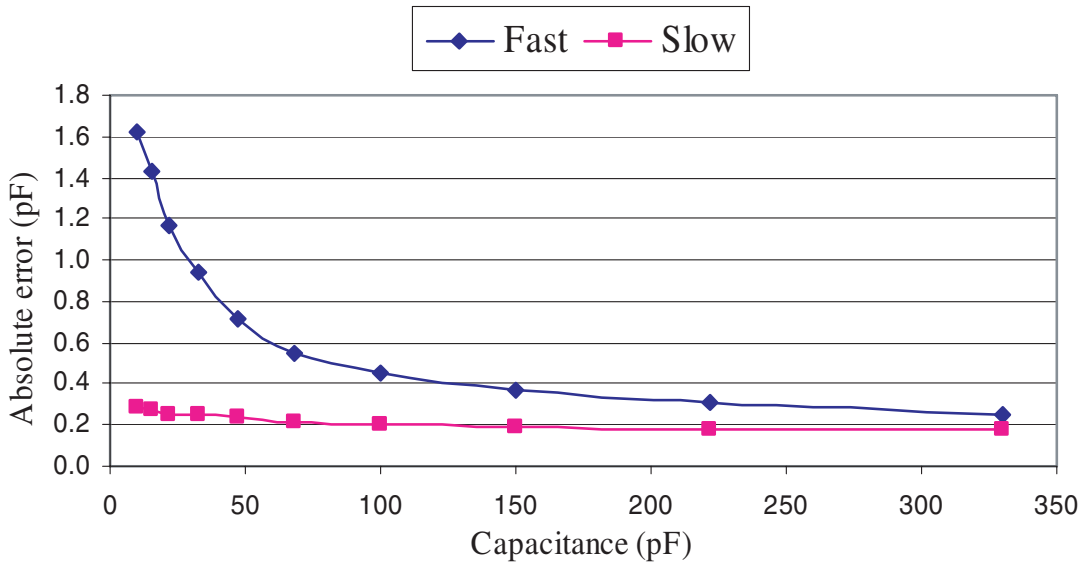

Figure 11. Comparison of the measured absolute error versus $C_{x}$ in slow and fast modes for $30 \mathrm{~m}$ of the cable with a twisted ground wire.

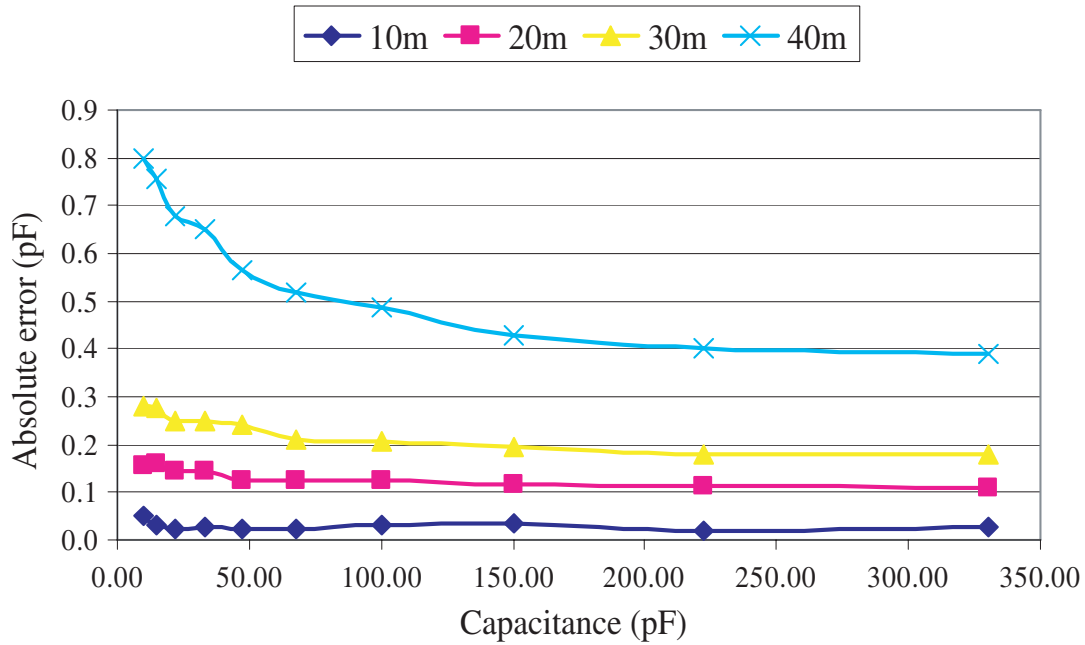

Figure 12. The measured absolute error versus $C_{x}$ for different lengths of the cable with a twisted ground wire.

Regarding the noise performance, it has been found that even for large values of the parasitic capacitance $C_{\mathrm{p} 1}$ and $C_{\mathrm{p} 2}$ (figure 4), these capacitances hardly affect the standard deviation. This shows that the noise performance is dominated by that of the voltage-to-period converter (figure 2) because otherwise, with increasing $C_{\mathrm{p} 1}$, due to the increased noise gain of amplifier $\mathrm{A}_{1}$ (figure 4(a)), the output noise should increase [10]. Figure 13 shows the measured results for 100 measurements of a capacitance with a nominal value of $330 \mathrm{pF}$, for a measurement time of $40 \mathrm{~ms}$ including three-signal autocalibration. The measured standard deviation amounts to $6.2 \mathrm{fF}$, which corresponds to about 16 bits of resolution. 


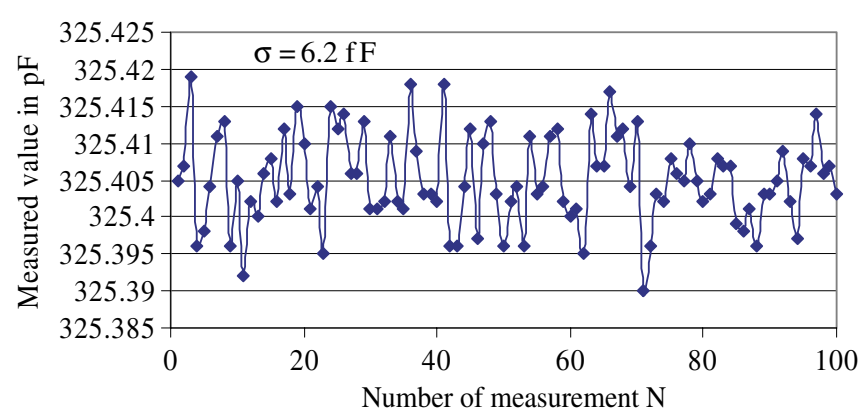

Figure 13. The measured value for a capacitor with a nominal value of $330 \mathrm{pF}$ for a measurement time of $40 \mathrm{~ms}$.

This result is about one bit better than that reported in [6] for comparable conditions.

We also measured the nonlinearity according to the method presented in [11]. In order to be independent of the absolute component accuracy, four different measurements are performed for $C_{\text {ref1 }}, C_{\text {ref2 }}, C_{\text {ref1 }}+C_{\text {ref3 }}$ and $C_{\text {ref2 }}+C_{\text {ref3 }}$, respectively. Supposing linear capacitance-to-time conversion $\left(T_{\mathrm{i}}=a C_{\mathrm{i}}+b\right)$, independent of the capacitance value, the value of the nonlinearity $\lambda$ as calculated by the equation

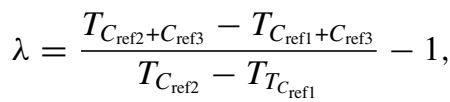

should be zero. Any deviation of $\lambda$ from zero can be defined as nonlinearity. The measurement of this nonlinearity should be performed in such a way that the parasitic capacitance (parasitic capacitances of PCB) remains constant during the four measurements. This means that not only the wiring of the setup but also its surrounding should not be changed [12]. In order to implement this, one common side of all three capacitors is connected to the interface, while the other side of these capacitors is connected to the ground or to the guarddrive voltage source.

In our nonlinearity tests, we selected different combinations of $C_{\text {ref1 }}, C_{\text {ref2 }}$, and $C_{\text {ref3 }}$ in such a way that $C_{\text {ref1 }}$, $C_{\text {ref2 }}, C_{\text {ref1 }}+C_{\text {ref3 }}$, and $C_{\text {ref2 }}+C_{\text {ref3 }}$ are within the range of $10 \mathrm{pF}$ to $330 \mathrm{pF}$, which resulted in a maximum measured nonlinearity of $3 \times 10^{-4}$.

\section{Conclusion}

An integrated version of switched-capacitor interface for a grounded capacitive sensor with feed-forward-based active shielding has been analyzed. The major nonidealities of the interface have been discussed. The complete interface has been designed and implemented using $0.7 \mu \mathrm{m}$ standard CMOS technology. The measurement results show good agreement with simulation results. It has been proven that, when we give the circuit enough time to settle, long connection cables can be used. Our measurements show that a capacitance as small as $10 \mathrm{pF}$ with $30 \mathrm{~m}$ of the connection cable can be measured with an error of less than $0.3 \mathrm{pF}$. For this length of cable, for the range of $C_{x}$ values from $10 \mathrm{pF}$ to $330 \mathrm{pF}$, the measured nonlinearity is less than $3 \times 10^{-4}$. With a measurement time of $40 \mathrm{~ms}$ a resolution of almost 16 bits has been found.

\section{Acknowledgments}

This work was supported in part by the Iranian Ministry of Science and Technology through a doctoral Grant, and by the Dutch technology Foundation (STW), The Netherlands, under project DET 6437.

\section{References}

[1] Huang S M, Stott A L, Green R G and Beck M S 1988 Electronic transducers for industrial measurement of low value capacitances J. Phys. E: Sci. Instrum. 21 242-50

[2] Joshi H, Vaghasiya K and Matthews T 2005 Dual-phase charged-based capacitance measurement technique Circuit Syst. 2 1000-2

[3] Baxter L K 1997 Capacitive sensors Design and Applications (New York: IEEE Press)

[4] Bera S C, Ray J K and Chattopadhayays S 2006 A low-cost non-contact capacitance-type level measurement for a conducting liquid IEEE Trans. Instrum. Meas. 55 778-86

[5] Reverter F, Li X and Meijer G C M 2006 Stability and accuracy of active shielding for grounded capacitive sensors Meas. Sci. Technol. 17 2884-90

[6] Reverter F, Li X and Meijer G C M 2008 A novel interface circuit for grounded capacitive sensors with feedforwardbased active shielding Meas. Sci. Technol. 19025202

[7] Goes F 1996 Low-cost smart sensor interfacing PhD Thesis Delft University of Technology, The Netherlands

[8] Goes F and Meijer G C M 1997 A universal transducer interface for capacitive and resistive sensor element Analog Integr. Circuits Signal Process. 14 249-60

[9] Sheu B J and Hu C 1984 Switched-induced error voltage on a switched capacitor IEEE J. Solid State Circuit SC-19 519-25

[10] Gasulla M, Li X and Meijer G C M 2005 The noise performance of a high-speed capacitive-sensor interface based on a relaxation oscillator and a fast counter IEEE Trans. Instrum. Meas. 54 1934-40

[11] Heidary A and Meijer G C M 2008 Features and design constraints for an optimised SC front-end circuit for capacitive sensors with a wide dynamic range J. Solid State Circuit 43 1609-16

[12] Heerens W C 1986 Review article application of capacitance techniques in sensor design J. Phys. E: Sci. Instrum. 19 897-906 